\title{
Uso de agrotóxicos e os riscos à saúde humana: panorama da área rural do município de Independência - RS
}

\author{
Use of pesticides and risks to human health: overview of the rural area of the municipality of \\ Independência - RS \\ Uso de plaguicidas y riesgos para la salud humana: visión general del área rural del municipio de \\ Independência - RS
}

Recebido: 28/09/2021 | Revisado: 10/10/2021 | Aceito: 16/10/2021 | Publicado: 18/10/2021

Fernanda Meller
ORCID: https://orcid.org/0000-0001-7196-8447
Sociedade Educacional Três de Maio, Brasil
E-mail: fernanda-meller@ hotmail.com
Angélica Reolon-Costa
ORCID: https://orcid.org/0000-0002-8160-9373
Sociedade Educacional Três de Maio, Brasil
E-mail: angelicacosta@ setrem.com.br
Silvana Ceolin
ORCID: https://orcid.org/0000-0001-6635-5515
Sociedade Educacional Três de Maio, Brasil
E-mail: silvanaceolin@ setrem.com.br

\begin{abstract}
Resumo
O Objetivo geral estudo foi descrever os distúrbios de saúde e os aspectos relativos ao uso de agrotóxicos por trabalhadores rurais do município de Independência-RS. Para tal, desenvolveu-se uma pesquisa de caráter quantitativo do tipo descritivo exploratória, que foi realizada no município de Independência - Rio Grande do Sul, em uma amostra de 63 agricultores. Os dados foram coletados por meio de um formulário com questões objetivas, no período de novembro de 2019 a fevereiro de 2020. As análises dos resultados basearam-se na análise estatística descritiva. Os agricultores possuem conhecimento dos riscos associados ao uso de agrotóxicos, e já apresentaram distúrbios na saúde associados à exposição a tais produtos como, náusea, diarreia, irritação na pele, ardência nos olhos, porém não tiveram diagnóstico de intoxicação. Os fatores de risco associados a ocorrência de distúrbios na saúde por exposição aos agrotóxicos no município de Independência-RS são a baixa escolaridade, o não uso adequado dos equipamentos de proteção individual, uso de princípios ativos com grau variado de toxicidade, incluindo alguns classificados como cancerígenos, e a inexistência de depósito adequado para armazenamento dos agrotóxicos. Pesquisas como essas podem auxiliar na promoção de saúde dos trabalhadores rurais, prevenindo assim o número de intoxicações e agravos a saúde humana.
\end{abstract}

Palavras-chave: Agrotóxicos; Trabalhadores rurais; Risco á saúde.

\begin{abstract}
The general objective of the study was to describe health disorders and aspects related to the use of pesticides by rural workers in the municipality of Independência-RS. To this end, a quantitative research of the exploratory descriptive type was developed, which was carried out in the municipality of Independência - Rio Grande do Sul, in a sample of 63 farmers. Data were collected through a form with objective questions, from November 2019 to February 2020. The analysis of the results was based on descriptive statistical analysis. Farmers are aware of the risks associated with the use of pesticides, and have already presented health disorders associated with exposure to such products, such as nausea, diarrhea, skin irritation, burning eyes, but they have not had a diagnosis of intoxication. The risk factors associated with the occurrence of health disorders due to exposure to pesticides in the municipality of IndependênciaRS are low education, the inadequate use of personal protective equipment, use of active ingredients with varying degrees of toxicity, including some classified as carcinogens, and the lack of adequate storage for pesticides. Researches such as these can help to promote the health of rural workers, thus preventing the number of poisonings and harm to human health.
\end{abstract}

Keywords: Pesticides; Rural workers; Health risk.

\section{Resumen}

El objetivo general del estudio fue describir los trastornos de salud y aspectos relacionados con el uso de plaguicidas por parte de los trabajadores rurales del municipio de Independência-RS. Para ello, se desarrolló una investigación 
cuantitativa de tipo exploratorio descriptivo, que se llevó a cabo en el municipio de Independência - Rio Grande do Sul, en una muestra de 63 agricultores. Los datos fueron recolectados a través de un formulario con preguntas objetivas, desde noviembre de 2019 hasta febrero de 2020. El análisis de los resultados se basó en análisis estadístico descriptivo. Los agricultores son conscientes de los riesgos asociados al uso de plaguicidas, y ya han presentado trastornos de salud asociados a la exposición a dichos productos, como náuseas, diarrea, irritación de la piel, ardor de ojos, pero no han tenido un diagnóstico de intoxicación. Los factores de riesgo asociados a la ocurrencia de trastornos de salud por exposición a plaguicidas en el municipio de Independência-RS son baja educación, uso inadecuado de equipo de protección personal, uso de ingredientes activos con diversos grados de toxicidad, incluidos algunos clasificados como cancerígenos, y la falta de almacenamiento adecuado de plaguicidas. Investigaciones como estas pueden ayudar a promover la salud de los trabajadores rurales, evitando así el número de intoxicaciones y daños a la salud humana.

Palabras clave: Plaguicidas; Trabajadores rurales; Riesgo de salud.

\section{Introdução}

A partir do ano de 1950 nos Estados Unidos iniciou-se a utilização de agrotóxicos em grande escala, este movimento ficou marcado como 'Revolução Verde', que teve por objetivo modernizar a agricultura e aumentar produtividade. No Brasil esse movimento chegou na década de 1960, com a implantação do Programa Nacional de Defensivos Agrícolas (PNDA) (Embrapa, 2018). Esses fatos impulsionaram o desenvolvimento de novos agrotóxicos e a utilização em larga escala desses produtos, em prejuízo à saúde coletiva.

Neste contexto, o Brasil se solidificou como um dos maiores consumidores de agrotóxicos a nível mundial. O uso desenfreado desses produtos, tem desencadeado impactos na saúde do homem e no meio ambiente (Barbosa et al., 2014; Pignati et al., 2017). De acordo com a Organização Mundial da Saúde, os agrotóxicos constituem um risco para a saúde devido a exposições únicas ou múltiplas durante o manuseio (Cézar-Vaz et al., 2016; Cabral \& Alonzo, 2019). Os seus efeitos diretos e indiretos, sejam imediatos ou de médio a longo prazo, preocupam cientistas, profissionais da área da saúde, ambientalistas e a sociedade em geral há décadas (Souza et al., 2017).

O uso de agrotóxicos pode resultar em cefaleia, êmese, vertigens, irritação dos olhos e na pele. Além de insônia, anemia, alterações hormonais, problemas imunológicos, infertilidade, malformações congênitas e abortos (Dutra \& Souza, 2017; Santos et al., 2018; Taveria \& Albuquerque, 2018; Lopes \& Albuquerque, 2019; Pereira et al., 2019). Está associado também à incidência de câncer, como linfoma e leucemia, e a ocorrência de alteração nos genes do embrião/feto em formação, resultando em malformações dos sistemas, endócrino, reprodutivo, neurológico, imunológico (Pereira et al., 2019; Leão et al., 2018). Destaca-se ainda que os quadros de intoxicação podem ser leve, moderada ou grave, dependendo da quantidade absorvida, do tempo de absorção, da toxicidade e princípio ativo do produto (Lopes \& Albuquerque, 2018).

No Brasil, 61,1\% dos casos de pessoas expostas a agrotóxicos tiveram diagnóstico de intoxicação confirmado, 89\% evoluíram para cura, 2,4\% para cura com sequela e 0,5\% para óbito (Brasil, 2020). No Rio Grande Do Sul, em 2007 foram registrados 69 casos, com observado aumento de 259 casos em 2015 (Brasil, 2020; Costa et al., 2016; Brasil, 2018). No mesmo ano, o referido estado registrou 47 hospitalizações por agrotóxicos domésticos, 9 internações decorrentes de exposição a raticidas e, 5 casos de hospitalização por uso de produtos veterinários (Costa et al., 2016; Brasil, 2018).

Estes dados demostram que a exposição aos agrotóxicos se constitui num problema de saúde pública (Dutra \& Souza, 2017; Santos et al., 2018; Taveria \& Albuquerque, 2018; Lopes \& Albuquerque, 2019). Destaca-se que mesmo tendo conhecimentos dos riscos associados ao uso de agrotóxicos, muitos agricultores não fazem o uso correto dos equipamentos de proteção individual, armazenam os produtos em local inadequado, queimando ou enterrando embalagens vazias, negligenciando os efeitos sobre a saúde e ambiente (Santos et al., 2018; Menegat et al., 2019). A exposição direta aos agrotóxicos aponta a necessidade de medidas para redução dos riscos associados a sua utilização, sendo necessário que os profissionais de saúde compreendam que o trabalho é um importante fator determinante da saúde (Menegat et al., 2019).

Neste contexto, emerge a necessidade de conscientização sobre os agravos associados a exposição aos agrotóxicos, da 
importância do manejo adequado como forma de prevenção de intoxicações e de distúrbios na saúde, bem como, do desenvolvimento e implementação de ações em saúde com tal finalidade. Ressalta-se ainda, que ao considerar que o município de estudo tem a sua economia baseada na agricultura, torna-se relevante pesquisas que analisam os distúrbios de saúde e outros aspectos relativos o uso de agrotóxicos por trabalhadores rurais.

Em vista disso, o presente estudo assume um papel primordial durante as atividades assistenciais dos profissionais de saúde sobretudo enfermeiros, visando conhecer a territorialização local, sobretudo voltando um olhar crítico as pessoas susceptíveis, proporcionando uma educação continuada sobre a importância do manejo adequado dos agrotóxicos na prevenção dos riscos á saúde do trabalhador.

Diante disso, busca-se responder a seguinte problemática: quais os fatores de risco e os distúrbios da saúde estão associados ao uso de agrotóxicos por trabalhadores rurais do município de Independência-RS? Sendo o objetivo do estudo: descrever os distúrbios de saúde e os aspectos relativos ao uso de agrotóxicos por trabalhadores rurais do município de Independência-RS

\section{Metodologia}

A pesquisa é de abordagem quantitativa, do tipo descritivo exploratório, que foi realizada na área rural do município de Independência, Rio Grande do Sul (RS). A coleta de dados ocorreu durante no período de novembro de 2019 a fevereiro de 2020. Conforme o Censo demográfico de 2010 do IBGE (2010) a população do município de Independência/RS é de 6.618 habitantes e teve população estimada para 2017 de 6.598 habitantes. A estrutura fundiária do município consta de 31.510 hectares, distribuídos em 1.335 propriedades.

Foram incluídos neste estudo agricultores que residem no referido município, que possuem entre 50 a 100 hectares de área cultivada, trabalham na atividade agrícola e são maiores de 18 anos. Assim a população de serviu de base, para o cálculo amostral foi constituída de setenta agricultores. Sobre esta foi calculado o tamanho amostral para planejamento da média numérica de pessoas, que deveriam ser entrevistadas, sendo utilizada a fórmula proposta por Santos (2016):

Quadro 1. Fórmula de cálculo amostral.

$$
n=\frac{N \cdot Z^{2} \cdot p \cdot(1-p)}{Z^{2} \cdot p \cdot(1-p)+e^{2} \cdot(N-1)}
$$

Legenda: $\mathrm{n}$ - amostra calculada / N - população / Z - variável padronizada associada ao nível de confiança / p - verdadeira probabilidade do evento / e - erro amostral

Fonte: Santos (2016) adaptado por Meller, Reolon-Costa, Ceolin (2019).

Nesta base de cálculo se obteve uma amostra de 63 agricultores residentes no Independência, levando em consideração um nível de confiança de $90 \%$ do cálculo e um erro amostral de 5\%.

A coleta de dados foi realizada por meio da aplicação de um formulário com questões objetivas, para caracterização do perfil sociodemográfico, além do levantamento de dados acerca do conhecimento dos agricultores sobre os riscos de intoxicações, princípios ativos e classe toxicológica, uso de equipamentos de proteção, sinais e sintomas, destino e armazenamento das embalagens.

A pesquisa foi realizada na residência dos entrevistados. O pesquisador após se apresentar explicou o objetivo, entregando o Termo de Consentimento Livre Esclarecido, o qual o entrevistado ao aceitar participar da pesquisa assinou e recebeu uma cópia do mesmo. Em seguida, o participante respondeu ao formulário acima proposto. Não houve recusa de nenhum dos participantes em participar da pesquisa, nem perda de amostra. 
Nos entrevistados que relataram apresentar mais de cinco sinais e sintomas relacionados ao uso de agrotóxicos foi realizado o exame de Colinesterase Plasmática que serve para verificar envenenamento ou o grau de exposição da pessoa a produtos tóxicos. Os valores de referência para homens é de 5.826 à $16.246 \mathrm{U} / \mathrm{L}$ e mulheres é de 4.782 à 15.186 U/L.

Os participantes que aceitaram fazer o exame foram acompanhados pelos responsáveis pelo projeto, até o Laboratório KL Análises Clínicas conveniado a Hermes Pardini de Minas Gerais. A coleta de sangue foi realizada pelo Técnico responsável, 24 horas após os mesmos realizarem atividades de aplicação de agrotóxicos. As despesas referentes aos exames foram de responsabilidade dos pesquisadores.

Os dados foram analisados pelo uso da estatística descritiva simples, foi realizado o cálculo das frequências absoluta, relativa e relativa acumulada, com o auxílio do software Microsoft Excel. A apresentação dos dados foi feita em tabelas. A pesquisa foi submetida ao Comitê de Ética e Pesquisa (CEP) da Universidade de Passo de Fundo (UPF), no Rio Grande do Sul (RS), obtendo parecer favorável para a coleta de dados sob Certificado de Apresentação para Apreciação Ética (CAAE) $n^{\circ}$ 98055418.4.0000.5342

Os materiais com as informações obtidas para realização da pesquisa incluíram um Termo de Consentimento Livre e Esclarecido, conforme preconiza a Resolução nº 466/12 do Conselho Nacional de Saúde/Ministério da Saúde (CNS/MS).

\section{Resultados}

Observou-se que a maioria dos entrevistados tem idade acima de 46 anos, é do sexo masculino e possui Ensino Fundamental incompleto (Tabela 1). Além disso, entra em contato direto com agrotóxicos periodicamente e faz a leitura dos rótulos de orientações contidas nesses produtos (Tabela 1).

Tabela 1. Características sociodemográficas dos trabalhadores rurais entrevistados, Independência - RS, 2021.

\begin{tabular}{llll}
\hline Variáveis & FA & FR (\%) & FRA (\%) \\
\hline Idade & & & \\
\hline 20- 35 anos & 14 & 22 & 22 \\
36-45 anos & 06 & 9 & 31 \\
46-65 anos & 35 & 56 & 87 \\
Acima de 65 anos & 08 & 13 & 100 \\
\hline Escolaridade & & & \\
\hline Ensino fundamental incompleto & 32 & 51 & 51 \\
Ensino fundamental completo & 06 & 9 & 60 \\
Ensino médio incompleto & 02 & 3 & 63 \\
Ensino médio completo & 15 & 24 & 87 \\
Ensino superior & 08 & 13 & 100 \\
\hline Residência & & & \\
\hline Área rural & 63 & 100 & 100 \\
Área urbana & 0 & 0 & 100 \\
\hline Faz uso de agrotóxicos & & & \\
\hline Sim & 63 & 100 & 100 \\
Não & 0 & 0 & 100 \\
Leitura das orientações dos rótulos e bulas & & & \\
Sim & 34 & 54 & 54 \\
Não & 29 & 46 & 100 \\
\hline
\end{tabular}

Legenda: Frequência Absoluta (FA), Frequência relativa (FR), Frequência relativa acumulada (FRA)

Fonte: Autores (2021).

Os participantes relataram que o princípio ativo mais utilizado nas propriedades é o Glifosato, seguido do Paraquat e 2-4 D Amina. Também informaram que os agrotóxicos utilizados pertencem as seguintes classes toxicológicas: mediamente 
tóxico (cor azul), extremamente tóxico (cor vermelha) e altamente tóxico (cor amarela) (Tabela 2), sendo a classificação toxicológica usada neste estudo anterior a reclassificação proposta de Resolução da ANVISA RDC n. 294 de 29 de julho de 2019.

Tabela 2. Conhecimento dos trabalhadores rurais quanto aos agrotóxicos, intoxicações e uso de EPIs, Independência - RS, 2021.

\begin{tabular}{|c|c|c|c|}
\hline Variável & FA & FR (\%) & FRA (\%) \\
\hline \multicolumn{4}{|l|}{ Princípios ativos mais utilizados } \\
\hline Piretróides & 15 & 24 & 24 \\
\hline Organofosforados & 20 & 32 & 56 \\
\hline Glifosato & 63 & 100 & 156 \\
\hline Atrazina & 34 & 54 & 210 \\
\hline Tiametozam & 10 & 16 & 226 \\
\hline Carbozina & 10 & 16 & 242 \\
\hline Paraquat & 54 & 86 & 328 \\
\hline Abamectina & 27 & 43 & 371 \\
\hline Acefato & 24 & 38 & 409 \\
\hline Lefenuron & 5 & 8 & 417 \\
\hline 2-4 D amina & 43 & 68 & 485 \\
\hline Clorantraniliprole & 1 & 2 & 487 \\
\hline \multicolumn{4}{|l|}{ Classe toxicológica } \\
\hline vermelho- extremamente tóxico & 46 & 73 & 73 \\
\hline azul- mediamente tóxico & 59 & 94 & 167 \\
\hline amarelo- altamente tóxico & 41 & 65 & 232 \\
\hline verde- pouco tóxico & 30 & 48 & 280 \\
\hline \multicolumn{4}{|l|}{ Periodicidade } \\
\hline Diário & 0 & 0 & 0 \\
\hline Semanal & 1 & 2 & 2 \\
\hline Quinzenal & 41 & 65 & 67 \\
\hline Mensal & 21 & 33 & 100 \\
\hline \multicolumn{4}{|l|}{ Uso de EPI'S } \\
\hline Sim & 31 & 49 & 49 \\
\hline Não & 33 & 52 & 100 \\
\hline \multicolumn{4}{|l|}{ Se sim quais? } \\
\hline Luvas & 19 & 30 & 30 \\
\hline Botas & 24 & 38 & 68 \\
\hline Óculos & 7 & 11 & 79 \\
\hline Máscara & 23 & 36 & 115 \\
\hline Roupas de proteção & 17 & 27 & 142 \\
\hline Outras & 1 & 2 & 144 \\
\hline \multicolumn{4}{|l|}{ Uso trator gabinado para a aplicação } \\
\hline Sim & 21 & 33 & 33 \\
\hline Não & 42 & 67 & 100 \\
\hline
\end{tabular}

Legenda: Frequência Absoluta (FA), Frequência relativa (FR), Frequência relativa acumulada (FRA)

Fonte: Autores (2021).

Com relação à periodicidade do uso de agrotóxicos, a maioria faz uso quinzenalmente e durante ciclo da cultura como forma de controle de pragas e doenças na lavoura. Outros ainda entram em contato com agrotóxicos mensalmente, semanalmente e, ocasionalmente, para reparos diversos. 
Com relação aos equipamentos de proteção individual (EPIs) a maior porcentagem da amostra diz não fazer o uso. No entanto, os agricultores que fazem uso, o fazem de forma inadequada, optando por um ou outro acessório, como botas, máscaras e luvas (Tabela 2). Dentre os 63 agricultores investigados, $42 \%$ disseram não fazer a pulverização com trator gabinado, sendo este fato um fator de risco, considerando que os agricultores por não usarem EPIs de forma adequada ficam completamente expostos aos agrotóxicos (Tabela 2).

Sobre o local de estocagem dos agrotóxicos ainda em uso, a maioria mantém no galpão, onde são armazenados outros produtos, como sementes, maquinário agrícola, rações, forma de armazenamento considerada não adequada (Tabela 3).

Tabela 3. Estocagem e destino das embalagens, sintomas após ou durante a aplicação e diagnóstico. Independência - RS, 2021.

\begin{tabular}{|c|c|c|c|}
\hline Variáveis & FA & FR (\%) & FRC (\%) \\
\hline \multicolumn{4}{|l|}{ Depósito dos agrotóxicos ainda em utilização } \\
\hline Casa & 0 & 0 & 0 \\
\hline Galpão & 51 & 81 & 81 \\
\hline Lugar exclusivo & 11 & 18 & 99 \\
\hline Outros & 01 & 1 & 100 \\
\hline \multicolumn{4}{|l|}{ Armazenagem das embalagens vazias } \\
\hline Casa & 0 & 0 & 0 \\
\hline Galpão & 18 & 29 & 29 \\
\hline Queimadas & 13 & 21 & 50 \\
\hline Enterradas & 0 & 0 & 50 \\
\hline Devolvidas no estabelecimento de compra & 32 & 50 & 100 \\
\hline \multicolumn{4}{|l|}{ Distúrbios na saúde } \\
\hline Perda de peso & 0 & 0 & 0 \\
\hline Irritabilidade & 3 & 5 & 5 \\
\hline Diarreia & 7 & 11 & 16 \\
\hline Alergias na pele & 9 & 14 & 30 \\
\hline Fraqueza muscular & 2 & 3 & 33 \\
\hline Insônia & 0 & 0 & 33 \\
\hline Náusea & 6 & 10 & 43 \\
\hline Tosse & 0 & 0 & 43 \\
\hline Depressão & 2 & 3 & 46 \\
\hline Ardência nos olhos & 24 & 38 & 84 \\
\hline Vômito & 2 & 3 & 87 \\
\hline Problemas respiratórios & 1 & 2 & 89 \\
\hline \multicolumn{4}{|l|}{ Diagnóstico de intoxicação } \\
\hline Sim & 6 & 10 & 10 \\
\hline Não & 57 & 90 & 100 \\
\hline \multicolumn{4}{|l|}{ Se sim - serviço de saúde estava vinculado } \\
\hline Publica (SUS) & 1 & 3,17 & 3,17 \\
\hline Privado & 5 & 7,93 & 11,1 \\
\hline
\end{tabular}

Legenda: Frequência Absoluta (FA), Frequência relativa (FR), Frequência relativa acumulada (FRA)

Fonte: Autores (2021).

Em relação ao destino final das embalagens, 50\% dos agricultores devolvem ao fornecedor do produto, estando de acordo com a Lei $\mathrm{n}^{\circ} 9.974$ de 06 de junho de 2000 (Tabela 3). Mesmo que a maioria dos entrevistados destine de forma correta, uma parcela significativa queima ou deixa guardado no galpão (Tabela 3) o que representa risco ambiental e para a saúde.

A maior parte da amostra analisada nunca realizou exames para diagnóstico de intoxicação, mesmo já tendo apresentando algum distúrbio na saúde associado. Os distúrbios evidenciados, durante ou após o manejo dos agrotóxicos foram, ardência nos olhos e alergias na pele, diarreia, náusea, irritabilidade, vômito e fraqueza muscular (Tabela 3). Entre aqueles agricultores que foram diagnosticados com intoxicação por agrotóxicos, a maioria estava vinculada a rede privada de 
saúde.

Os resultados das atividades das colinesterases plasmáticas encontraram-se dentro dos limites biológicos de referência (5.826 à 16.246 U/L) (Figura 1).

Figura 1. Níveis de Colinesterase Plasmática, em dez participantes do estudo. Independência - RS, 2021.

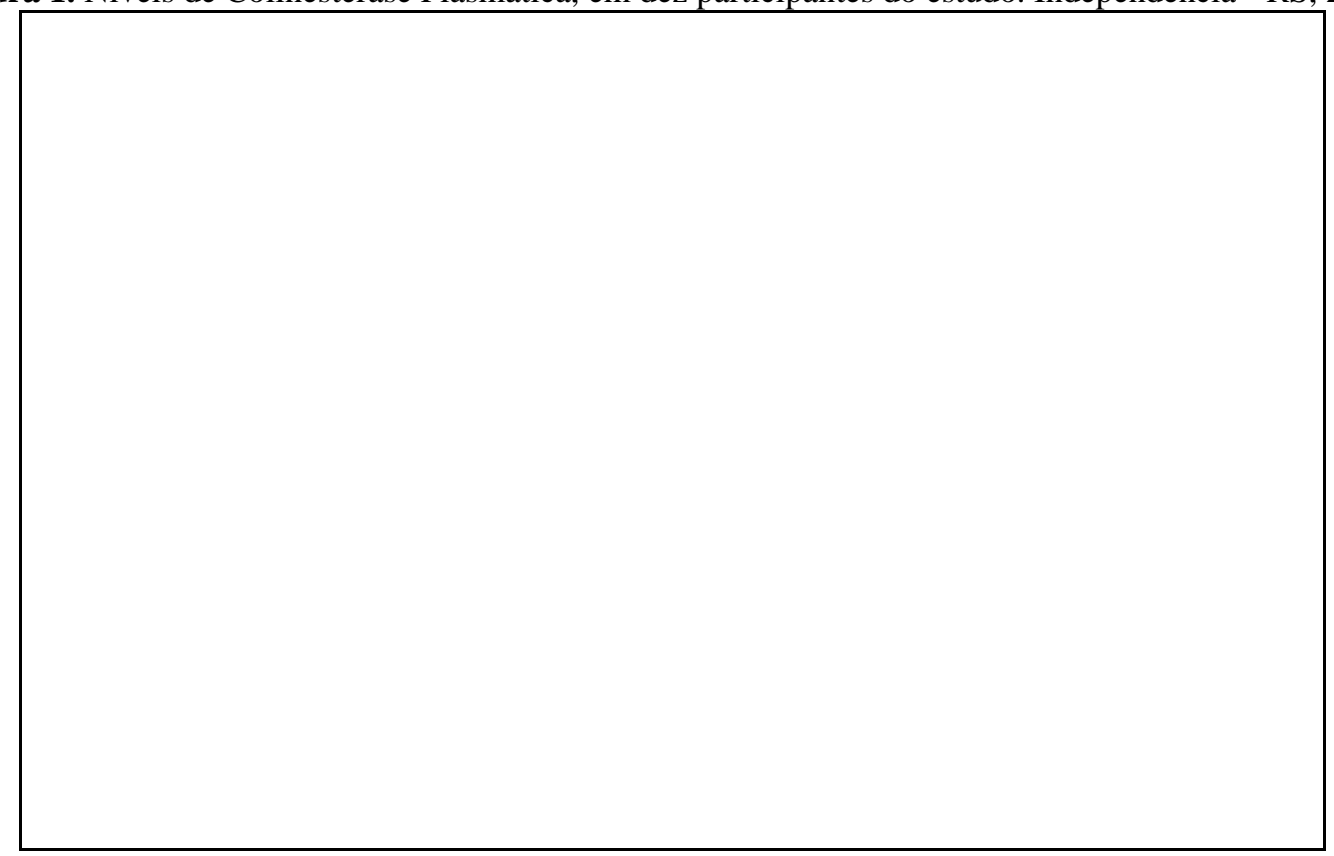

Fonte: Autores (2021).

Conforme demostrado na figura 1 apenas um dos dez participantes apresentou nível de colinesterase abaixo do valor de referência (4.986 U/L) o que indica intoxicação por exposição aguda por organofosforados, outros dois agricultores o valor esteve próximo do mínimo aceitável (6.926 U/L e 8.394 U/L). No participante que apresentou nível de intoxicação por exposição aguda a agrotóxicos, foi orientado que o mesmo procurasse um serviço de saúde para fazer um tratamento, conforme a orientação médica.

\section{Discussão}

Os agricultores têm conhecimento dos riscos relacionados ao consumo e manuseio de agrotóxicos. Sendo os distúrbios na saúde relatados pelos mesmos, náusea, vômito, tontura, ardência nos olhos e/ou irritação na pele, durante e após a aplicação. Além disso, a maioria possui baixa escolaridade, não faz uso adequado dos equipamentos de proteção individual, fazem uso de princípios ativos com grau variado de toxicidade, incluindo alguns classificados como cancerígenos ao ser humano, além de, não possuírem depósito adequado para armazenamento dos agrotóxicos, sendo esses os fatores de risco associados a possiblidade de ocorrência de distúrbios na saúde por exposição aos agrotóxicos observados na amostra estudada.

A atividade de aplicação e manipulação de agrotóxicos é predominante no sexo masculino, estando esses indivíduos frequentemente expostos ao risco de intoxicação (Menegat et al., 2019). Fato que fica evidente quando se analisam os índices de intoxicações distribuídos por gênero no Brasil, no Rio Grande do Sul e na Região Noroeste do referido estado, no período de 2013 a 2020, no qual se observa que maioria dos casos notificados são em homens (Brasil, 2020, Costa et al, 2016; Brasil, 2018). Corroborando com estudo desenvolvido por Cabral \& Alonzo (2019) no Estado de Alagoas, nos anos de 2010, 2011, 2012 e 2015, o qual observou que maioria dos casos de intoxicação registrados eram em homens, sendo apenas 3\% em mulheres. 
No entanto, é importante a conscientização dos riscos associados a exposição aos agrotóxicos em homens e mulheres. Segundo Leão et al. (2019) e Santa et al. (2016) essas realizam atividades de aplicação desses produtos em hortaliças e pomares, além da lavagem de roupas contaminadas, da manipulação de embalagens e, por estarem próximas as áreas de aplicação, estando sujeitas a deriva técnica e, portanto, aos riscos de exposição ambiental. A dificuldade de mensurar o impacto da exposição feminina, ocorre em parte, porque o trabalho rural muitas vezes não é considerado por elas como um trabalho, mas como ajuda aos seus parceiros (Santa et al., 2016).

A baixa escolaridade observada, pode ser considerada um fator de risco de intoxicações. Pois dificulta a leitura e entendimento das informações técnicas presentes nos rótulos e bulas dos produtos, bem como, da importância da utilização adequada de EPIs durante a aplicação (Lopes \& Albuquerque, 2018; Pereira et al., 2019; Carneiro et al., 2015). Por outro lado, os agricultores não devem ser culpabilizados pela baixa escolaridade e consequente intoxicação. Sobre isto, a legislação prevê̂ que, à direita do eixo central da faixa colorida dos rótulos de agrotóxicos, sejam colocados pictogramas referentes às precauções durante a aplicação, que devem ser de fácil entendimento, o que seria uma alternativa para as pessoas de baixa escolaridade.

Entre os princípios ativos mais utilizados pelos agricultores está o glifosato, produto usado na dessecação de plantas invasoras. Conforme Hass et al. (2018), no ser humano, esse produto pode inibir processos metabólicos das bactérias do trato gastrointestinal de quem o ingere. Outros herbicidas como as acetamidas e o 2,4 D (ácido diclorofenoxiacético) também são usados. Exposições ocupacionais ao 2,4-D podem ocorrer durante a fabricação e aplicação, enquanto a população em geral pode ser exposta por meio de alimentos, água, poeira, aplicação residencial e durante a pulverização. Em humanos, o 2,4-D é eliminado praticamente inalterado na urina (Loomis et al., 2015).

Ainda segundo Loomis et al. (2015) e Guyton et al. (2015) o glifosato e o 2,4 D podem causar corrosão na pele e irritação ocular, náusea, cefaleia tontura, vômito, desorientação e dificuldade respiratória. Porém o principal problema decorrente da exposição a esses agrotóxicos, são a ocorrência de intoxicações crônicas, resultando desregulação endócrina, malformações e alguns distúrbios mentais. Além disso, estão classificados como provavelmente carcinogênicos para seres humanos conforme o relatório divulgado pela Agência Internacional de Pesquisas do Câncer. Experimentos realizados por Loomis et al. (2015) em células in vitro e in vivo evidenciaram a influência do 2,4 D nos mecanismos celulares, resultando em estresse oxidativo e problemas no sistema imunossupressor, bem como, sua relação com a ocorrência de linfoma e leucemia. Já testes realizados por Guyton et al. (2015) com glifosato apresentaram tendência positiva na incidência de carcinoma do túbulo renal, hemangiossarcoma, adenoma de células das ilhotas pancreáticas e tumores de pele.

Outros produtos usados entre os agricultores, são os organofosforados, que possuem grau variado de toxicidade, e que podem provocar sudorese, distúrbios gastrintestinais, e no sistema nervoso, miose, taquicardia, arritmias, fraqueza, convulsões e morte (Inouyea et al., 2014).

Nesse estudo, a classe toxicológica mediamente tóxica foi relatada pelos agricultores como mais usada, dado que não é compatível com os princípios ativos mais usados, demonstrando a falta de conhecimento dos produtores sobre a classificação toxicológica dos agrotóxicos. Importante destacar que um agrotóxico de faixa vermelha traz risco mesmo quando a exposição é pequena. No entanto, um de faixa verde também traz risco se a exposição a ele for grande, isto é, se o organismo for exposto a ele por longo tempo, ou a altas doses (Silva et al., 2017).

O levantamento da periodicidade do uso de agrotóxicos é necessário para determinar os riscos de exposição a intoxicações dos agricultores. É preocupante o observado nesse trabalho, pois os produtores ficam expostos a esses produtos praticamente todo o ano, devido as demandas da produção agrícola.

Quanto aos EPIs, o não uso ou uso inadequado é prática recorrente entre agricultores. Estudos feitos por Costa e Pires (2016), Pertali et al. (2019) e Cargnin et al., (2017) relatam que o não uso dos equipamentos de proteção individual está 
associado principalmente ao desconforto térmico. Os autores afirmam ainda que, como esses equipamentos são projetados e os materiais utilizados na confecção afetam os mecanismos de termorregulação corporal, o que pode ser agravado, pela intensa exposição solar e elevado esforço físico decorrentes do trabalho no campo. O uso inadequado de EPIs, também foi relatado em trabalho desenvolvido com 100 fumicultores da região noroeste do Rio Grande do Sul, onde os produtores afirmaram fazer uso apenas de botas, luvas, óculos, chapéu ou macacão, devido ao desconforto térmico e a dificuldade na execução do trabalho (Cargnin et al., 2017 e Alves, 2017).

Outro aspecto evidenciado nesse estudo é de que os produtores não possuem depósito adequado para os produtos ainda não usados, ou em uso. Fato que aumenta os riscos de exposição, ocupacional e acidental. O mesmo foi observado por Santana et al. (2019) e Lopes (2016) os relatam que a maioria dos casos o armazamento é feito fora de casa, em galpões junto com outros produtos, incluindo aqueles destinados a alimentação animal. Quanto ao descarte das embalagens, a maioria dos agricultores cumpre com a Lei $\mathrm{n}^{\circ} 9.974$ de 06 de junho de 2000, que dispõe sobre a produção, a embalagem e a rotulagem, o transporte, o armazenamento, a comercialização, a utilização e a fiscalização de agrotóxicos, devolvendo essas embalagens aos fornecedores, porém alguns produtores compram os produtos em local inadequado (Brasil, 2000).

Os mesmos distúrbios na saúde por exposição aos agrotóxicos relatados neste estudo, foram observados em outras pesquisas, sendo esses, dor de cabeça, enjoo, diminuição da visão, vertigem, irritação da pele, perda de apetite, tremores, vômito, crise alérgica, diarreia, dores no peito, secura na garganta e nervosismo (Rocha \& Oliveira; 2016; Pertali et al., 2019; Alvez, 2017; Santana et al., 2019); Alves, 2016; Cunha, 2017).

A apesar de uma minoria ter sido diagnosticada com intoxicação por agrotóxicos, esse aspecto não deixa de ser preocupante, uma vez que existe um número significativo de casos que não são diagnosticados ou notificados corretamente. Outro aspecto relevante é que mesmo com o diagnóstico de intoxicação os agricultores continuam nas atividades agrícolas habituais. Como observado em pesquisa realizada no do Vale do São Francisco, onde maior parte dos pesquisados teve diagnóstico positivo de intoxicação, e permanecem em suas atividades (Araújo \& Oliveira, 2017).

Neste cenário, salienta-se a importância da interferência do enfermeiro na prevenção de intoxicações e de distúrbios da saúde associados aos agrotóxicos. O profissional da enfermagem pode oferecer a direção que é essencial ao domínio dos casos de intoxicação, devendo, conforme as condições da tarefa especializada, ofertar um auxílio e a sustentação que dele espontaneamente se aguarda. O enfermeiro deve ficar vigilante as cautelas destinadas às ocorrências de exposição e contaminação por agrotóxicos, tomando pra si a sensatez de guardar pela saúde e a melhor qualidade de vida do trabalhador quando em seu meio de atividade (Cunha, 2017).

Destaca-se ainda que o baixo índice de casos de intoxicação observado, pode estar associado ao não diagnóstico, diagnóstico incorreto e a subnotificação. O que pode estar associado ao despreparo dos profissionais de saúde e ao fato dos trabalhadores não procurem o atendimento mesmo quando possuem algum sinal ou sintoma relacionado (Carneiro et al., 2015). Desta forma é necessário investir na formação e qualificação dos profissionais na enfermagem, bem como, no estímulo ao registro/notificação das ocorrências nos sistemas de informação em saúde do SUS (Santana et al., 2016).

O diagnóstico de intoxicação pode ser realizado através da análise sanguínea pelo exame de colinesterase, nesse estudo dos onze agricultores submetidos ao exame, apenas um foi diagnosticado com intoxicação. Já em estudo realizado com agricultores de Minas Gerais, constatou-se através de análise sanguínea que $50 \%$ dos trabalhadores estavam intoxicados (Oliveira et al., 2018).

Com base do supracitado é evidente a necessidade de uma maior abordagem sobre a utilização de agrotóxicos por meio de palestras, oficinas, ou outras atividades que possam minimizar os riscos a que os agricultores estão submetidos. Neste contexto, os profissionais da enfermagem podem autuar no desenvolvimento de práticas de cuidado e gestão aos grupos populacionais com risco de exposição aos agrotóxicos. 
Tal atuação, é preponderante no desenvolvimento de ações de conscientização e de difusão de informação entre os agricultores, além do fornecimento das orientações, bem como, a assistência, o suporte e os cuidados necessários nos casos de intoxicação por agrotóxicos, contribuindo assim para a redução da ocorrência de casos de intoxicação por exposição a esses agentes químicos. Como limitante deste estudo encontram-se o fato do exame de colinesterase ter sido realizado em uma pequena amostra de agricultores.

\section{Conclusão}

Os resultados do estudo apontam que os agricultores têm conhecimento de que há riscos relacionados ao consumo e manuseio de agrotóxicos, manifestando preocupação com as consequências disso. Nesse contexto os fatores de risco associados a possiblidade de ocorrência de distúrbios na saúde por exposição aos agrotóxicos evidenciados nesse estudo são: a baixa escolaridade, não uso e uso inadequado de EPIs, princípios ativos, classe toxicológica dos produtos usados, desconhecimento da classe toxicológica dos produtos usados, bem como, o armazamento incorreto desses.

A relação entre o uso de agrotóxicos e a ocorrência de distúrbio a saúde, fica evidente uma vez que os produtores relataram sentir sinais e sintomas, associados a tais distúrbios como náusea, vômito, tontura, ardência nos olhos ou irritação na pele, durante a após a aplicação. Este fato é reforçado, pelo número de produtores que já foram diagnosticados com intoxicação, e pelo resultado do exame de colinesterase, onde um dos produtores analisados apresentou valores a baixo do nível de referência indicando intoxicação por organofosforados.

Sugere-se ainda a realização de novos estudos que objetivem a realizam do exame de colinesterase em uma amostra maior de agricultores, o que pode contribui para a obtenção de resultados que reflitam significativamente a realidade da população estudada.

\section{Referências}

Alves, D. Ações de saúde a produtores de tabaco expostos a agrotóxicos: estudo em um município de atividade econômica predominantemente agrícola. (2017). [Trabalho de conclusão de curso]. Santa Cruz do Sul (RS). Curso Bacharelado em Enfermagem. Universidade de Santa Cruz do Sul - USCS.

Araújo, I. M. M., \& Oliveira, A. G. R. C. (2017). Agronegócio e agrotóxicos: impactos à saúde dos trabalhadores agrícolas no nordeste brasileiro. Trabalho, Educação e Saúde, 15(1):117-129. https://doi.org/10.1590/1981-7746-sol00043.

Barbosa, R. S., Silveira, H. F., Cerqueira, G. S., Júnior, H. L. R., Carvalho, S. S., \& Alves, G. C. S. (2014). Exposição Ocupacional aos Agrotóxicos: Um Estudo Bibliográfico. Revi Inter. Toxic. Risco Ambiental. Soc., 7(1): 50-61. https://doi.org/10.22280/revintervol7ed1.166

Brasil. Lei $\mathrm{n}^{\circ} .9 .974$, de 6 de junho de 2000. (2000). Dispõe sobre a produção, destino, embalagens, transporte e armazenamento de agrotóxicos. Brasil. Diário Oficial da União, República Federativa do Brasil. Seção 1. http://www.planalto.gov.br/ccivil_03/leis/19974.htm

Cabral, E. R. M., \& Alonzo, H. G. A. (2019). Aumento das exposições aos agrotóxicos: contribuição da enfermagem. Rev. Enfer. Atual. Inder, 87(2):87-8. https://doi.org/10.31011/reaid-2019-v.87-n.25-art.207

Cargnin, M. C. S., Echer, I. C \& Silva, D. R. (2017). Fumicultura: uso de equipamento de proteção individual e intoxicação por agrotóxico. Rev. cuidado é fundamental, 9(2): 466-472. http://dx.doi.org/10.9789/2175-5361.2017.v9i2.466-472

Carneiro, F. F., Augusto, L. G. S., Rigotto, R. M., Friedrich K., \& Campos A. (0215). Dossiê ABRASCO: um alerta sobre os impactos dos agrotóxicos na saúde. Fiocruz, 1(1): 1-616. https://www.abrasco.org.br/site/outras-noticias/institucional/dossie-abrasco-um-alerta-sobre-os-impactos-dos-agrotoxicos-nasaude/9898/.

Cezar-Vaz, M. R., Bonow, C. A., Mello, M. C. V. A., \& Silva, M. R. S. (2016). Abordagem socioambiental na enfermagem: focalizando o trabalho rural e uso de agrotóxicos. Rev. Bras. Enferm, 69(6): 1114-21. https://doi.org/10.1590/0034-7167-2016-0364

Costa E. T., Soares I. C., Michel R. S \& Garibotti V. (2016). Cenário da Intoxicação por Agrotóxicos no Rio Grande do Sul. Bol. Epidemiológico, 18(2):1-16. https://cevs.rs.gov.br/upload/arquivos/201706/12111248-be-v18-n12-2016-internet.pdf

Costa L. F \& Pires G. L. P. (2016). Análise histórica sobre a agricultura e o advento do uso de agrotóxicos no brasil. Rev. Ciênc, 12(12):1-17. http://intertemas.toledoprudente.edu.br/index.php/ETIC/article/view/5433

Cunha, N. A. (2017). Acidentes de trabalho com exposição a material biológico: análise epidemiológica e percepção das vítimas, em Uberlândia - MG. [Dissertação]. Ubelândia (SP). Curso de Mestrado Profissional em Saúde Ambiental e Saúde do Trabalhador. Instituto de Geografia. 
Dutra R. M.S \& Suza M. M. O. (2017). Impactos negativos do uso de agrotóxicos à saúde humana. Rev. Bras. de Geografia Médica e da Saúde, 13(24): 127140. http://www.seer.ufu.br/index.php/hygeia/article/view/34540

EMBRAPA. Empresa Brasileira de Pesquisa Agropecuária. (2018). Soja em números. https://www.embrapa.br/soja/cultivos/soja1/dados-economicos .

Guyton K., Loomis D., Grosse Y., Ghissassi F., Tallaa L. B \& Guha N. (2015). Carcinogenicity of tetrachlorvinphos, parathion, malathion, diazinon, and glyphosate. Rev. The Lancet Oncology, 16(5): 490-491. https://doi.org/10.1016/S1470-2045(15)70134-8

Hass P., Hoehne L \& Kuhn D. (2018). Revisão: avaliação dos efeitos do glifosato no ecossistema agrícola e sua toxicidade para a saúde humana. Rev. Destaques Acadêmicos, 10(4): 82-90. http://www.univates.br/revistas/index.php/destaques/article/view/2014/1447

Inouyea L. A., Fernandez L. M., Carneiro L. F. S., Germano J. J \& Criscib A. R. (2014). Avaliação Morfologica do Fígado e do Pulmão Pós Intoxicação por Organofosforado, em Ratos Wistar. Rev. Uniciências, 18(2): 103-109. https://doi.org/10.17921/1415-5141.2014v18n2p\%25p

Leão R. S., Marques R. C., Buralli R. J., Silva, D. S \& Guimarães, G. R. D. Avaliação da saúde pública exposição a agroquímicos: uma experiência com a agricultura familiar o noroeste do Rio de Janeiro. Rev. Susten. em debate, 9(1):81-94. https://doi.org/10.18472/SustDeb.v9n1.2018.26956

Loomis D., Guyton K., Grosse Y., Ghissasi F., Bouvard, V., \& Tallaa L. B. (2015). Carcinogenicity of lindane, DDT, and 2,4-dichlorophenoxyacetic acid. Rev. The Lancet Oncology, 16(8): 891-892. //doi.org/10.1016/S1470-2045(15)00081-9

Lopes C. V. A \& Albuquerque G. S. C. (2018). Agrotóxicos e seus impactos na saúde humana e ambiental: uma revisão sistemática. Saúde em debate, 42(117): 518-534. https://doi.org/10.1590/0103-1104201811714.

Lopes F. G. (2016). Avaliação do conhecimento dos agricultores (as) quanto aos riscos dos agrotóxicos e os cuidados com o seu uso. Cadernos de Agroecologia, 10(3):1-5. http://revistas.aba-agroecologia.org.br/index.php/cad/article/view/17757

Menegat B., Reolon-Costa A \& Caramão G. S. Conhecimento dos agricultores sobre riscos de intoxicação pelo uso de agrotóxicos. Ciência, Cuidado e Saúde, 18(2): 2-7]. https://doi.org/10.4025/cienccuidsaude.v18i2.39659

Ministério da Saúde. Intoxicações exógenas relacionadas ao trabalho no Brasil, 2007-2016. (2020). Bol. Epidemiológico, 49(58): 1-45. http://portalarquivos2.saude.gov.br/images/pdf/2018/dezembro/26/2018-027.pdf

Oliveira F. M., Silva G. M. N., Lima A. S., Santos K. P. P., Batista W. F. M., \& Barros R. F. M. (2018). Agrotóxicos: impactos sobre o meio ambiente e saúde dos agricultores na comunidade Graciosa / José de Freitas / PI / Brasil. Anais do VI Congresso Latino-americano de Agroecologia, X Congresso Brasileiro de Agroecologia, V Seminário de Agroecologia do Distrito Federal e Entorno. Brasília/DF. 2018 18(1).

Pereira R. A., Costa R. A \& Lima E. M. (2019). O impacto dos agrotóxicos sobre a saúde humana e o meio ambiente. Extensão, 3(1):29-37. https://revista.unitins.br/index.php/extensao/article/view/1684/1122.

Petarli G. B., Cattafesta M., Luz T. C., Zandonade E, Bezerra O. M. P. A., \& Salaroli L. B. (2019). Exposição ocupacional a agrotóxicos, riscos e práticas de segurança na agricultura familiar no município do Espírito Santo. Brasil. Rev. Bras. Saúde Ocupacional, 44(15): 1-13. https://doi.org/10.1590/23176369000030418

Pignati W. A., Lima F. A. N. S., Lara S. S., Correa M. L. M., Barbosa J. R., Leão L. H. C., \& Pignatti, M. G. (2017). Distribuição espacial do uso de agrotóxicos no Brasil: uma ferramenta para a Vigilância em Saúde. Rev. Ciênc. Saúde Coletiva, 22(10): 3281-3293. https://doi.org/10.1590/1413812320172210.17742017 .

Rocha T. A. L. C. G., \& Oliveira F. N (2016). Occupational Safety and Health: Vulnerability and perception of risks related to the use of agrochemicals in an irrigated horticulture center of Rio Grande do Norte. Rev. Gestão, Produção e Transformação, 23(3): 600-611. http://dx.doi.org/10.1590/0104-530x1219-14

Santana C. M., Costa A. R., Nunes R. M. P., Peron A. P., Cavalcante A. A.C. M \& Ferreira P. M. P. (2016). Exposição ocupacional de trabalhadores rurais a agrotóxicos. Cad. Saúde colet, 24(3): 301-307. https://doi.org/10.1590/1414-462x201600030199.

Santana V. T. P., Duarte P. M., \& Dalmas A. D. (2019). Perfil das vítimas intoxicadas por agrotóxicos no estado do Rio Grande do Sul entre o período de 2007 a 2017. Rev. Ciênc. Biol. e da saúde, 25(1): 147-163. https://www.researchgate.net/deref/http\%3A\%2F\%2Fdx.doi.org\%2F10.18312\%2F19807341.n21.2019.1404

Santos, V. C. F., Trevilato, G. C., Mesquita, M. O., Ruiz, E. M. F., Riquinho, D. L \& Meneget, R. P. (2018). O uso de agrotóxicos e suas implicações para a saúde humana e ambiental: enfrentamento local. Rev. Lume, 1(1):181-195. https://www.lume.ufrgs.br/bitstream/handle/10183/1 84491/001079419.pdf?sequence=

Silva J. V., Vilela L. P., Moraes, M. S., \& Silveira, C. A. (2017). A percepção dos trabalhadores rurais sobre a autoexposição aos agrotóxicos. Rev. Saúde, 43(1): 199-205. https://doi.org/10.5902/2236583422163

Souza M. M. T., Vargas F. R., Costa P. S., Tosoli A. M. G., Balbino C. M., \&Passos, J. P. (2017). A trabalhadora rural e a exposição ocupacional no cultivo do tomate. Rev. Enfer. Atual, 88(26): 1-10. https://doi.org/10.31011/reaid-2019-v.88-n.26-art.2

Taveira, B. L. S \& Albuquerque, G. S. C. (2018). Análise das notificações de intoxicações agudas, por agrotóxicos, em 38 municípios do estado do Paraná. Rev. Saúde em Debate, 42(4): 211-222. https://doi.org/10.1590/0103-11042018s417 